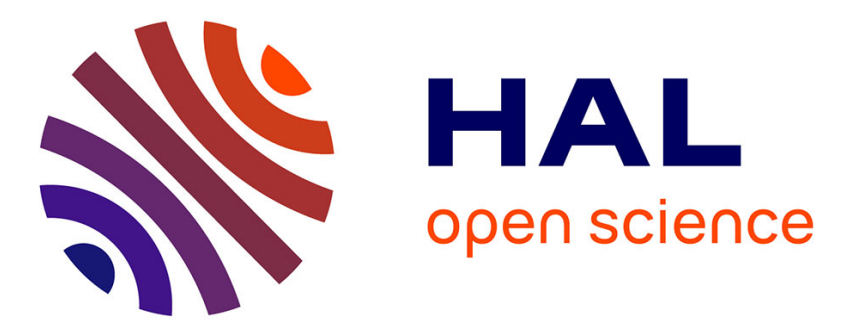

\title{
Using a high-gain observer for a hybrid output feedback: finite-time and asymptotic cases for SISO affine systems
}

Swann Marx, Vincent Andrieu, Christophe Prieur

\section{To cite this version:}

Swann Marx, Vincent Andrieu, Christophe Prieur. Using a high-gain observer for a hybrid output feedback: finite-time and asymptotic cases for SISO affine systems. ACC 2014 - American Control Conference, Jun 2014, Portland, United States. pp.n/c. hal-00997236

\section{HAL Id: hal-00997236 https://hal.science/hal-00997236}

Submitted on 27 May 2014

HAL is a multi-disciplinary open access archive for the deposit and dissemination of scientific research documents, whether they are published or not. The documents may come from teaching and research institutions in France or abroad, or from public or private research centers.
L'archive ouverte pluridisciplinaire HAL, est destinée au dépôt et à la diffusion de documents scientifiques de niveau recherche, publiés ou non, émanant des établissements d'enseignement et de recherche français ou étrangers, des laboratoires publics ou privés. 


\title{
Using a high-gain observer for a hybrid output feedback: finite-time and asymptotic cases for SISO affine systems
}

\author{
Swann Marx ${ }^{1}$, Vincent Andrieu ${ }^{2}$ and Christophe Prieur ${ }^{3}$
}

\begin{abstract}
This article suggests a design method of hybrid output feedbacks for affine systems under observability and stabilizability assumptions. Our aim is to use the separation principle on systems controlled by hybrid feedback laws. We investigate two constructive methods for the high-gain observer: the first one is based on a finite-time convergence of the observation error, the second one is based on an asymptotic convergence of the observation error. We illustrate one of our main results on a well-known example: integrators chain.
\end{abstract}

\section{INTRODUCTION}

In recent decades, many methods have been introduced for designing output feedback laws that asymptotically stabilizes the origin of a nonlinear system (see e.g [15], [1], [2], [4]). More recently, thanks to the hybrid formalism described in [8], new methods have been introduced to design asymptotically stabilizing hybrid state feedbacks laws. This allows to consider a larger class of system (for instance the Brockett integrator [5]). Moreover, hybrid state feedbacks laws may increase performances (see for instance [12]).

The design of output feedback controllers may be obtained from an observer and a state feedback design. Note however that for nonlinear continuous systems, designing separately each of these tools leads only to local result. Following [15], when the observer is tuned based on the robustness property of the continuous state feedback, a semi-global result may be achieved. In this paper, we extend this approach to the case in which the state feedback is hybrid.

This paper is organized as follows. In Section III by considering stabilizability and observability assumptions, a hybrid output feedback law is designed by considering a high-gain observer which converges in finite-time. Such observers are based on the homogeneity notion (see e.g. [3]). This type of design may imply numerical problems. In Section [III by considering different stabilizability and observability assumptions that are stronger, we etablish a second theorem that deals with a more classical high-gain observer, because it converges asymptotically. This result is

\footnotetext{
${ }^{1}$ Swann Marx is a student from the École Normale Supérieure de Cachan, Electrical Engineering Department, 61 Avenue du Président Wilson, 94230 Cachan, France. This work began during an internship at Gipsa-lab, Grenoble, France and has been completed during an internship at the Universidad Técnica Fédérica Santa Marìa, Valparaìso, Chile. swann.marx@ens-cachan.fr.

${ }^{2}$ Vincent Andrieu is with Université Lyon 1 CNRS UMR 5007 LAGEP, France. vincent. andrieu@gmail.com.

${ }^{3}$ Christophe Prieur is with Department of Automatic Control, Gipsa-lab, 961 rue de la Houille Blanche, BP 46, 38402 Grenoble Cedex, France, christophe.prieur@gipsa-lab.fr.

This work has been partially supported by the ANR project LimICoS contract number 12 BS03 00501.

This work has been partially supported by CONICYT grant ACT-1106.
}

illustrated by the design of an output feedback controller for an integrators chain. Section IV collects some concluding remarks. Finally the appendix collects the proofs of the main results.

Due to space limitation, some poofs are omitted.

\section{First SET OF ASSUMPTIONS: The FINITE-TIME CASE}

Let us consider the single-input single-output system:

$$
\begin{aligned}
\dot{x}_{p} & =f_{p}\left(x_{p}\right)+g_{p}\left(x_{p}\right) u \\
y & =h_{p}\left(x_{p}, u\right),
\end{aligned}
$$

where $x_{p} \in \mathbb{R}^{n_{p}}, y \in \mathbb{R}, u \in \mathbb{R}, f_{p}: \mathbb{R}^{n_{p}} \rightarrow \mathbb{R}^{n_{p}}, g_{p}$ : $\mathbb{R}^{n_{p}} \rightarrow \mathbb{R}^{n_{p}}$ and $h_{p}: \mathbb{R}^{n_{p}} \times \mathbb{R} \rightarrow \mathbb{R}$ are locally Lipschitz functions. We assume that the origin is an equilibrium point for (1).

\section{A. Stabilizability assumption}

Consider the following nonlinear hybrid system $\mathcal{H}:=$ $(\mathcal{F}, F, \mathcal{J}, G)$ :

$$
\mathcal{H} \begin{cases}\dot{x} & =F(x), \quad x \in \mathcal{F} \\ x^{+}=G(x), & x \in \mathcal{J}\end{cases}
$$

where $\mathcal{F} \subset \mathbb{R}^{n}$ and $\mathcal{J} \subset \mathbb{R}^{n}$ are called respectively the flow and jump sets associated to the continuous and discrete dynamics given respectively by $F: \mathbb{R}^{n} \rightarrow \mathbb{R}^{n}$ and $G: \mathbb{R}^{n} \rightarrow$ $\mathbb{R}^{n}$. Given a closed set $\mathcal{A}$ and denoting $|\cdot|_{\mathcal{A}}$ the distance to $\mathcal{A}$, let us recall the following definition borrowed from $[8$, Definition 3.6]

Definition 1 (Uniform stability concepts).

- The set $\mathcal{A}$ is uniformly globally stable (UGS) for (2) if there exists a class $\mathcal{K}_{\infty}$ function $\alpha$ such that any solution $x$ to (2) satisfies $|x(t, j)|_{\mathcal{A}} \leq \alpha\left(|x(0,0)|_{\mathcal{A}}\right)$, for all $(t, j) \in \operatorname{dom}(x)$; - the set $\mathcal{A}$ is uniformly globally attractive (UGA) for (2) if for each $\varepsilon>0$ and $r>0$, there exists $T>0$ such that for any solution $x$ to (2) such that $|x(0,0)|_{\mathcal{A}} \leq r$ is complete and satisfies $|x(t, j)|_{\mathcal{A}} \leq \varepsilon, \forall(t, j) \in \operatorname{dom}(x), t+j \geq T$;

- the set $\mathcal{A}$ is uniformly globally asymptotically stable (UGAS) for (2) if it is both uniformly globally stable and uniformly globally attractive;

- given a set $\Gamma$, the $\mathcal{A}$ is uniformly semi-globally asymptotically stable with respect to $\Gamma$ for (2) if $\mathcal{A}$ is uniformly globally asymptotically stable, by considering only initial conditions in $\Gamma$.

Inspired by [14] and [13], we assume that the origin of (1) can be stabilized by a hybrid state feedback law.

Assumption 1 (Stabilizability). There exists a hybrid controller defined by $\left(\mathcal{F}_{c}, \mathcal{J}_{c}, f_{c}, g_{c}, \theta_{c}\right)$, where $\mathcal{F}_{c}$ and $\mathcal{J}_{c}$ are 
closed sets, $\mathcal{F}_{c} \cup \mathcal{J}_{c}=\mathbb{R}^{n_{p}+n_{c}}, g_{c}: \mathbb{R}^{n_{p}+n_{c}} \rightarrow \mathbb{R}^{n_{c}}$, $f_{c}: \mathbb{R}^{n_{p}+n_{c}} \rightarrow \mathbb{R}^{n_{c}}$ and $\theta_{c}: \mathbb{R}^{n_{p}+n_{c}} \rightarrow \mathbb{R}$ are continuous functions, such that the origin of the system:

$$
\begin{aligned}
& \left\{\begin{array}{l}
\dot{x}_{p}=f_{p}\left(x_{p}\right)+g_{p}\left(x_{p}\right) \theta_{c}\left(x_{p}, x_{c}\right) \\
\dot{x}_{c}=f_{c}\left(x_{p}, x_{c}\right)
\end{array} \quad\left(x_{p}, x_{c}\right) \in \mathcal{F}_{c}\right.
\end{aligned}
$$

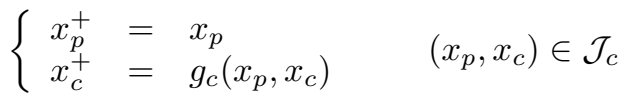

is uniformly globally asymptotically stable.

\section{B. Observability assumption}

Following [4], we define recursively the following functions for all $\left(x_{p}, \tilde{v}\right) \in \mathbb{R}^{n_{p}} \times \mathbb{R}^{n_{p}}$,

$$
\begin{aligned}
\varphi_{0}\left(x_{p}, v_{0}\right) & =h_{p}\left(x_{p}, v_{0}\right), \\
\varphi_{i}\left(x_{p}, v_{0}, \ldots, v_{i}\right) & =\frac{\partial \varphi_{i-1}}{\partial x_{p}} f_{p}\left(x_{p}, v_{0}\right)+\sum_{k=0}^{i-1} \frac{\partial \varphi_{i-1}}{\partial v_{k}} v_{k+1} .
\end{aligned}
$$

for all $i=1, \ldots, n_{p}-1$, where the notation $\tilde{v}=$ $\left(v_{0}, \ldots, v_{n_{p}-1}\right)$ has been used. We consider also the function $\phi_{c}: \mathbb{R}^{n_{p}} \times \mathbb{R}^{n_{p}} \rightarrow \mathbb{R}^{n_{p}}$ defined as

$$
\phi_{c}\left(x_{p}, \tilde{v}\right)=\left(\begin{array}{c}
\varphi_{0}\left(x_{p}, v_{0}\right) \\
\vdots \\
\varphi_{n_{p}-1}\left(x_{p}, v_{0}, \ldots, v_{n_{p}-1}\right)
\end{array}\right)
$$

Given a smooth function $u:[0, \infty) \rightarrow \mathbb{R}$, we denote, for all $x_{p} \in \mathbb{R}^{n_{p}}$ and $t \geq 0$,

$$
\phi\left(x_{p}, t ; u\right)=\phi_{c}\left(x_{p}, u(t), \ldots, u^{\left(n_{p}-1\right)}(t)\right) .
$$

where, for each $k \in \mathbb{N}, u^{(k)}$ denotes the $k$-th derivative of the function $u$. We can now state the observability assumption employed in the first main result:

Assumption 2 (Observability for a suitable controller). There exists a smooth controller $\bar{u}:[0, \infty) \rightarrow \mathbb{R}$, such that:

(i) For all $t \geq 0$, the function $x \mapsto \phi(x, t ; \bar{u})$ is injective on $\mathbb{R}^{n_{p}}$;

(ii) For all $t \geq 0$ and for all $x_{p} \in \mathbb{R}^{n_{p}}$, the matrix $\frac{\partial \phi\left(x_{p}, t ; \bar{u}\right)}{\partial x_{p}}$ is invertible.

Remark 1. With this property, and given a compact set of initial condition, it is possible to design a finite time highgain observer. Indeed, if (1) satisfies Assumption 2, then for each $t \geq 0$, the function $\phi(\cdot, t ; \bar{u})$ is a diffeomorphism from $\mathbb{R}^{n_{p}}$ to $\mathbb{R}^{n_{p}}$. Inspired by [6], by setting $u=\bar{u}(t)$ and $\mathcal{Z}=$ $\phi\left(x_{p}, t ; \bar{u}\right)$, the system (1) can be rewritten as follows:

$$
\dot{\mathcal{Z}}=\mathcal{S Z}+B \delta(\mathcal{Z}, t)
$$

where $\mathcal{Z}=\left[\begin{array}{c}z_{p_{1}} \\ z_{p_{2}} \\ \vdots \\ z_{p_{n_{p}}}\end{array}\right], \mathcal{S}=\left[\begin{array}{ccccc}0 & 1 & 0 & \ldots & 0 \\ 0 & 0 & 1 & \ddots & 0 \\ \vdots & \vdots & \ddots & \ddots & \vdots \\ 0 & 0 & \ldots & \ldots & 1 \\ 0 & \ldots & \ldots & \ldots & 0\end{array}\right], B=$ $[0, \cdots, 0,1]^{T}$ and $\delta: \mathbb{R}^{n_{p}} \times[0, \infty) \rightarrow \mathbb{R}$ is a nonlinear continuously differentiable function. Following [3], for all compact set of initial conditions and for all $T>0$ it is possible to design an observer which converges in time $T$.
Under Assumptions 1 and 2, we are interested in the design of a hybrid output feedback law that makes the origin of the system (1) semi-globally asymptotically stable by coupling the state feedback considered in Assumption 1 and a finite-time high-gain observer that will be obtained from Assumption 2.

Coupling Assumptions 1 and 2 together with a temporal timer and a high-gain strategy as in [7] yields the following hybrid system:

$$
\begin{aligned}
& \left\{\begin{array}{l}
\dot{x}_{p}=f_{p}\left(x_{p}\right)+g_{p}\left(x_{p}\right) \mathcal{U}\left(\hat{x}_{p}, x_{c}, \sigma\right) \\
\dot{x}_{c}=f_{c}\left(\hat{x}_{p}, x_{c}\right) \\
\dot{\hat{x}}_{p}=\psi_{p}\left(\hat{x}_{p}, x_{c}, \sigma, \mathcal{U}, y\right) \\
\dot{\sigma}=s(\sigma) \\
\quad\left(\hat{x}_{p}, x_{c}, \sigma\right) \in \mathcal{F}_{c} \times[0, T]
\end{array}\right. \\
& \left\{\begin{array}{l}
x_{p}^{+}=x_{p} \\
x_{c}^{+}=g_{c}\left(\hat{x}_{p}, x_{c}\right) \\
\hat{x}_{p}^{+}=\hat{x}_{p} \\
\sigma^{+}=\sigma
\end{array} \quad\left(\hat{x}_{p}, x_{c}, \sigma\right) \in \mathcal{J}_{c} \times[T,+\infty)\right.
\end{aligned}
$$

where $\mathcal{U}=\mathcal{U}\left(\hat{x}_{p}, x_{c}, \sigma\right)$ is such that $\mathcal{U}=\bar{u}(\sigma)$ if $\sigma \leq T$ and $u=\theta_{c}\left(\hat{x}_{p}, x_{c}\right)$ if $\sigma>T, \psi_{p}$ is a continuous vector field to be designed, $\sigma$ stands for the timer state and $s:[0, \infty) \rightarrow$ $[0, \infty)$ is a smooth function such that $s(\sigma)=1$ for $\sigma \leq T$ and $s(2 T)=0$.

\section{First main result}

Theorem 1. (Attractivity for appropriate initial timer states) Under Assumptions 1 and 2 for all compact sets $\Gamma \subset \mathbb{R}^{n_{p}}$, there exist a positive real number $T$ and a function $\psi_{p}$ such that by focusing on solutions satisfying $\sigma(0)=0$, the origin of (5) is attractive with a basin of attraction containing $\Gamma \times\{0\}$. More precisely, for all $x^{\sharp} \in \Gamma$, the solutions of (5) starting from $\left(x_{p}(0), x_{c}(0), \hat{x}_{p}(0), \sigma(0)\right)=\left(x^{\sharp}, 0,0,0\right)$ converge to $\{0\} \times[0,2 T]$.

The proof of this result is omitted due to space limitation.

Remark 2. Let us emphasize that the property written in Theorem 1 is not the uniform semi-global asymptotic stability since only solutions of (5) with initial conditions satisfying $\sigma(0)=0$ are considered, and since we were not able to state the stability property.

Remark 3. By exploiting the high-gain observer and the timer, a convergence of the error observation is obtained in finite time. Roughly speaking, the designed output feedback controller first observes the state and then stabilizes it. Therefore this approach allows to exclude the Zeno solutions, and impose that the solutions follow a continuous time dynamics during $T$ units of time, so that the observer is able to converge.

\section{SECOND SET OF ASSUMPTIONS: THE ASYMPTOTIC CASE}

\section{A. Stabilizability Assumption}

In this section, we consider an approach similar to the one of [15]. Indeed, based on some hybrid stabilizability 
assumption and observability assumption, we obtain semiglobal asymptotic stabilizability of the origin. Note however that due to some particular effects of hybrid dynamics (for instance Zeno solutions) we require a persistent flow condition on the stabilizing state feedback.

Assumption 3 (Persistent flow stabilizability). There exist a hybrid controller $\left(\mathcal{F}_{c}, \mathcal{J}_{c}, f_{c}, g_{c}, \theta_{c}\right)$, a real $\lambda$ in $(0,1]$ such that the set $\{0\} \times[0,1]$ in $\mathbb{R}^{n_{p}+n_{c}} \times[0,1]$ is uniformly globally asymptotically stable for the following system

$$
\begin{gathered}
\left\{\begin{array}{l}
\dot{x}_{p}=f_{p}\left(x_{p}\right)+g_{p}\left(x_{p}\right) \theta_{c}\left(x_{p}, x_{c}\right) \\
\dot{x}_{c}=f_{c}\left(x_{p}, x_{c}\right) \\
\dot{\sigma}=1-\sigma \\
\left(x_{p}, x_{c}, \sigma\right) \in \mathcal{F}_{c} \times[0,+\infty),
\end{array}\right. \\
\left\{\begin{array}{l}
x_{p}^{+}=x_{p} \\
x_{c}^{+}=g_{c}\left(x_{p}, x_{c}\right) \quad\left(x_{p}, x_{c}, \sigma\right) \in \mathcal{J}_{c} \times[\lambda,+\infty) . \\
\sigma^{+}=0
\end{array}\right.
\end{gathered}
$$

Remark 4. This assumption is related to the notion of persistent flow, which charaterizes that a small dwell time $\lambda>0$ should exist between two successive jumps.

\section{B. Observability Assumption}

Moreover, in this context, we need an observability assumption uniform in the control input.

Assumption 4 (Complete Uniform Observability). System (1) is completely uniformly observable, that is

(i) For all $\tilde{v}$ in $\mathbb{R}^{n_{p}}$, the function $x_{p} \mapsto \phi_{c}\left(x_{p}, \tilde{v}\right)$ is injective on $\mathbb{R}^{n_{p}}$;

(ii) The matrix $\frac{\partial \phi_{c}\left(x_{p}, \tilde{v}\right)}{\partial x_{p}}$ is invertible for all $\left(x_{p}, \tilde{v}\right) \in$ $\mathbb{R}^{n_{p}} \times \mathbb{R}^{n_{p}}$.

\section{Second main result}

With the previous assumptions, it holds the following:

Theorem 2 (Semi-global asymptotic stability). Assume there exists a function $\gamma$ such that, for all $\left(x_{p}, x_{c}\right)$ in $\mathcal{J}_{c}$, we have:

$$
\left|g_{c}\left(x_{p}, x_{c}\right)\right| \leq \gamma\left(\left|x_{c}\right|\right)
$$

Under Asumptions 3 and 4 the origin of system (1) is uniformly semi-globally asymptotically stabilizable by a hybrid dynamic output feedback. More precisely, for all compact sets $\Gamma$ contained in $\mathbb{R}^{n_{p}}$, there exist a $C^{1}$ function $\Psi_{p}$ : $\mathbb{R}^{n_{p}} \times \mathbb{R} \times \mathbb{R} \rightarrow \mathbb{R}$ and a positive real number $c_{x}$ such that the set $\{0\} \times[0,1]$ in $\mathbb{R}^{2 n_{p}+n_{c}} \times[0,1]$ is uniformly asymptotically stable for the system

$$
\left\{\begin{aligned}
\dot{x}_{p} & =f_{p}\left(x_{p}\right)+g_{p}\left(x_{p}\right) u \\
\dot{\hat{x}}_{p} & =\Psi_{p}\left(\hat{x}_{p}, y, u\right) \\
\dot{x}_{c} & =f_{c}\left(\tilde{x}_{p}, x_{c}\right) \\
\dot{\sigma} & =1-\sigma \\
y & =h_{p}\left(\tilde{x}_{p}, u\right), u=\theta_{c}\left(\tilde{x}_{p}, x_{c}\right)
\end{aligned}\right.
$$

$$
\begin{aligned}
& \left(\tilde{x}_{p}, x_{c}, \sigma\right) \in \mathcal{F}_{c} \times[0,+\infty) \\
& \left\{\begin{array}{l}
x_{p}^{+}=x_{p} \\
\hat{x}_{p}^{+}=\hat{x}_{p} \\
x_{c}^{+}=g_{c}\left(\tilde{x}_{p}, x_{c}\right) \\
\sigma^{+}=0
\end{array} \quad\left(\tilde{x}_{p}, x_{c}, \sigma\right) \in \mathcal{J}_{c} \times[\lambda,+\infty) .\right.
\end{aligned}
$$

where 1

$$
\tilde{x}_{p}=\operatorname{Sat}_{c_{x}}\left(\hat{x}_{p}\right)
$$

with basin of attraction containing $\Gamma \times\{0\} \times \mathbb{R}_{+}$(which is a subset of $\mathbb{R}^{2 n_{p}+n_{c}} \times \mathbb{R}_{+}$).

Remark 5. Note that the timer avoids Zeno solutions. See e.g [10] and [9].

The main steps of the proof can be found in Appendix A

\section{Example: integrators chain}

We want to illustrate the Theorem 2 by applying it on the system $\dot{x}_{p_{1}}=x_{p_{2}}, \dot{x}_{p_{2}}=u+x_{p_{2}}^{2}$ and $y_{p}=x_{p_{1}}$, with the set $\Gamma=\left\{x_{p} \in \mathbb{R}^{2}: x_{p_{1}} \leq 50, x_{p_{2}} \leq 50\right\}$. Because this system has the same structure as (4), Assumption 4 holds.

We design a global controller: $u_{g}=-x_{p_{1}}-x_{p_{2}}^{2}-k_{1} x_{p_{2}}-$ $k_{2}\left(x_{p_{2}}+k_{1} x_{p_{1}}\right)$, where $k_{1}=1$ and $k_{2}=2$. We focus on the linearization around the origin and design a more efficient controller $\left(u_{l}=-K x_{p}\right.$, where $K$ is a matrix with appropriate dimensions and computed with a pole placement method, as described in e.g. [11]). By uniting these two controllers thanks to a discrete variable $x_{c} \in[0,1]$ (see e.g [12]) and setting $\lambda=0.01$, we get a hybrid controller that satisfies Assumption $3, \mathcal{F}_{c}$ and $\mathcal{J}_{c}$ are computed from the local version of the basin of attraction. Note that we can find a function $\gamma$ such that $\left|g_{c}\left(x_{p}, x_{c}\right)\right|:=\left|1-x_{c}\right| \leq \gamma\left(\left|x_{c}\right|\right)$

We tune the observer $\hat{x}_{p}=f_{p}\left(\hat{x}_{p}\right)+g_{p}\left(\hat{x}_{p}\right) \theta_{c}\left(\hat{x}_{p}, x_{c}\right)+$ $k\left(y_{p}, \hat{y}_{p}\right)$ where: $k\left(y_{p}, \hat{y}_{p}\right):=\left[\begin{array}{c}-280 \\ -2 * 280^{2}\end{array}\right]\left(y_{p}-\hat{y}_{p}\right)$.

Let us numerically compute the solutions whose initial conditions are $x_{p}^{\#}=(20,10)$ and $x_{c}^{\#}=0$. The phase portrait of the plant state and the time-evolution of $x_{c}$-variable are respectively given in Figures 1 and 2 . The stabilization is illustrated by Fig. 1. It is checked on Fig. 2 that there is no Zeno solution.

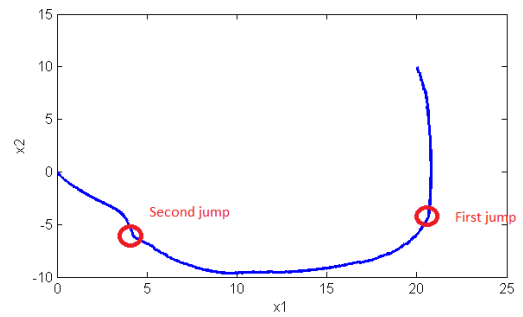

Fig. 1. Phase portrait of the plant state $x_{p}$. The two main jumps of the $x_{c}$-variable are marked by a circle

\footnotetext{
1 Given a positive real number $c$, Sat $c: \mathbb{R}^{n} \rightarrow \mathbb{R}^{n}$ is the saturating vector function defined by $\operatorname{Sat}_{c}(0)=0$ and $\operatorname{Sat}_{c}(x):=x \min \left\{1, \frac{c}{|x|}\right\}$, $\forall x \neq 0$.
} 


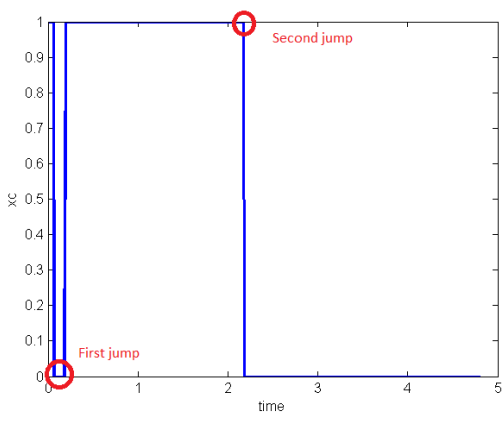

Fig. 2. Time-evolution of the $x_{c}$ variable. The two main jumps are marked by a circle.

\section{CONCLUSION}

In this paper, two techniques are proposed to design a hybrid output feedback controller. Both methods combine an observer and a hybrid stabilizing state feedback law. The first case considers a finite-time converging observer, whereas the second method suggests to use a high-gain controller asymptotically converging to the state. The asymptotic design is illustrated on a nonlinear control system.

This work lets different questions open. The first one is the relaxation of the persistent flow condition considered in Assumption 3 used in the second main result. This may be done by applying robustness arguments. Moreover the applications of these results to other nonlinear control systems are under actual investigation.

\section{APPENDIX}

\section{A. Proof of Theorem 2}

1) Lower bounded existence time in a compact set: This subsection is devoted to the tunning of the saturating parameter $c_{x}$ and to the construction of some sets. Indeed the construction of the observer is based on the construction of a specific set that needs to be selected in an appropriate way taking into account jumps that may occur due to the hybrid dynamics. Along this subsection, we consider the system defined by, for all $\left(x_{p}, x_{c}, \sigma\right)$ in $\left(\mathcal{F}_{c} \times \mathbb{R}_{+}\right) \cup\left(\mathcal{J}_{c} \times[\lambda,+\infty)\right)$,

$$
\begin{gathered}
\left\{\begin{array}{l}
\dot{x}_{p}=f_{p}\left(x_{p}\right)+g_{p}\left(x_{p}\right) \theta_{c}\left(\omega, x_{c}\right) \\
\dot{x}_{c}=f_{c}\left(\omega, x_{c}\right) \\
\dot{\sigma}=1-\sigma \\
\left(\omega, x_{c}, \sigma\right) \in \mathcal{F}_{c} \times[0,+\infty),
\end{array}\right. \\
\left\{\begin{array}{l}
x_{p}^{+}=x_{p} \\
x_{c}^{+}=g_{c}\left(\omega, x_{c}\right) \quad\left(\omega, x_{c}, \sigma\right) \in \mathcal{J}_{c} \times[\lambda,+\infty), \\
\sigma^{+}=0
\end{array}\right.
\end{gathered}
$$

where $\omega$ is an external time function in $\mathcal{L}_{\text {loc }}^{\infty}\left([0,+\infty) ; \mathbb{R}^{n_{p}}\right)$. Note that in the particular case where $\omega=\tilde{x}_{p}$, which is defined in (11), the solution to system (12)-(13) (without the dynamics $\left.\hat{x}_{p}\right)$ is also solution to system $(9)-(10)$.

To define the set of interest, consider $V: \mathbb{R}^{n_{p}+n_{c}+1} \rightarrow$ $\mathbb{R}_{+}$a continuously differentiable positive definite and proper Lyapunov function associated to the hybrid stabilizing state feedback of Assumption 3. Hence a real $\alpha$ in $(0,1)$ exists such that $V$ verifies:

$$
V\left(x_{p}, x_{c}, \sigma\right)=0 \Rightarrow x_{p}=0, x_{c}=0, \sigma \in[0,+\infty)
$$

and

$$
\begin{gathered}
\frac{\partial V}{\partial x}(x) F(x) \leq-V(x), \forall x \in \mathcal{F}_{c} \times[0,+\infty) \\
V(G(x))-V(x) \leq-\alpha V(x), \forall x \in \mathcal{J}_{c} \times[\lambda,+\infty)
\end{gathered}
$$

where we used the compact notation $x=\left(x_{p}, x_{c}, \sigma\right)$ and

$$
\begin{gathered}
F(x)=\left[\begin{array}{c}
f_{p}\left(x_{p}\right)+g_{p}\left(x_{p}\right) \theta_{c}\left(x_{p}, x_{c}\right) \\
f_{c}\left(x_{p}, x_{c}\right) \\
1-\sigma
\end{array}\right] \\
G(x)=\left[\begin{array}{c}
x_{p} \\
g_{c}\left(x_{p}, x_{c}\right) \\
0
\end{array}\right] .
\end{gathered}
$$

Let $m=\max _{x_{p} \in \Gamma, \sigma \in[0, \lambda]} V\left(x_{p}, 0, \sigma\right)$ and consider the following compact sets:

$$
\begin{aligned}
& D_{m}:=\left\{x \in \mathbb{R}^{n_{p}+n_{c}+1}, V(x) \leq m\right\}, \\
& D_{l_{1}}:=\left\{x \in \mathbb{R}^{n_{p}+n_{c}+1}, V(x) \leq l_{1}\right\},
\end{aligned}
$$

where $l_{1}>m$. Consider also the set

$D_{l_{1}}^{+}=\left\{\left(x_{p}, x_{c}, 0\right) \in \mathbb{R}^{n_{p}+n_{c}+1}\right.$,

$$
\left.\exists\left(x_{c}^{-}, \sigma^{-}\right) \in \mathbb{R}^{n_{c}+1},\left|x_{c}\right| \leq \gamma\left(\left|x_{c}^{-}\right|\right),\left(x_{p}, x_{c}^{-}, \sigma^{-}\right) \in D_{l_{1}}\right\} .
$$

We finally define the two last sets $D_{l_{2}}=\{x \in$ $\left.\mathbb{R}^{n_{p}+n_{c}+1}, V(x) \leq l_{2}\right\}$ and $D_{n}:=\left\{x \in \mathbb{R}^{n_{p}+n_{c}+1}, V(x) \leq\right.$ $n\}$ where $l_{2}>l_{1}$ is such that $D_{l_{1}}^{+} \subset D_{l_{2}}$ and where $n>l_{2}$. Let us now establish the following property for solutions of system (12)-(13) initiated from $D_{m}$.

Lemma 1. (Lower bounded existence time of solution in $\left.D_{n}\right)$ Let $c_{x}=\max _{\left(x_{p}, x_{c}, \sigma\right) \in D_{n}}\left\{\left|x_{p}\right|\right\}$. There exists $T_{\min }>0$ such that for all $\omega$ in $\mathcal{L}_{\text {loc }}^{\infty}\left([0,+\infty) ; \mathbb{R}^{n_{p}}\right)$ with $\omega(t) \leq c_{x}$ for all $t$ in $\left[0, T_{\min }\right]$, and all $x^{\#}:=\left(x_{p}^{\#}, x_{c}^{\#}, \sigma^{\#}\right)$ in $D_{m}$, all solutions $x(\cdot, \cdot)$ of 12$)-(13)$ with $x(0,0)=x^{\#}$ and all $(t, j)$ in $\operatorname{dom}(x)$ then $x(t, j) \in D_{n}$ for all $t \leq T_{\min }$.

Proof. Let $\bar{V}$ the positive real numbers defined by

$$
\bar{V}=\max _{x \in D_{n},|\omega| \leq c_{x}} \frac{\partial V}{\partial x}(x) F_{\omega}(x, \omega)
$$

where

$$
F_{\omega}(x, \omega)=\left[\begin{array}{c}
f_{p}\left(x_{p}\right)+g_{p}\left(x_{p}\right) \theta_{c}\left(\omega, x_{c}\right) \\
f_{c}\left(\omega, x_{c}\right) \\
1-\sigma
\end{array}\right] .
$$

In the remaining part of the proof, we show that Lemma 1 holds with $T_{\min }$ chosen as any positive real number satisfying

$$
T_{\min }<\inf \left\{-\ln (1-\lambda), \frac{l_{1}-m}{\bar{V}}, \frac{l_{2}-n}{\bar{V}}\right\} .
$$

Let $x^{\#}$ be in $D_{m}$ and let $x$ be a solution of system 12$)-(13)$ whose initial conditions are $x^{\#}$. For all $(t, j)$ in $\operatorname{dom}(x)$, we denote $\mathcal{V}(t, j)=V(x(t, j))$. 
Let $(t, j)$ in $\operatorname{dom}(x)$ such that $t \leq T_{\min }$. To prove the lemma, we need to show that $x(t, j)$ is in $D_{n}$. First of all, we show that $j \leq 1$. Indeed, assume $j \geq 2$. This implies that there exist $t_{0}$ and $t_{1}$ such that $0 \leq t_{0}<t_{1} \leq t$ such that $\left(t_{0}, 0\right),\left(t_{0}, 1\right),\left(t_{1}, 1\right),\left(t_{1}, 2\right)$ are in $\operatorname{dom}(x)$. Note that $\sigma\left(t_{0}, 1\right)=0$ and $\sigma\left(t_{1}, 1\right)=\lambda$. Moreover, for all $s$ in $\left[t_{0}, t_{1}\right]$, $(s, 1)$ is in $\operatorname{dom}(x)$ and that $\frac{\partial \sigma}{\partial t}(s, 1)=1-\sigma(s, 1)$. Hence, $t \geq t_{1}-t_{0}=-\ln (1-\lambda) \geq T_{\min }$. Hence this is impossible, and therefore $j \leq 1$.

So two cases may be distinguished.

$j=0$ This implies that $s \in[0, t] \mapsto x(s, 0)$ is a continuous mapping with $x(0,0)$ in $D_{n} \subset D_{l_{1}}$. Hence we can define $t^{*}$, the largest time in $[0, t]$ such that $x(s, 0)$ is in $D_{l_{1}}$ (i.e. $t^{*}=\max _{s \in[0, t], x(\ell, 0) \in D_{l_{1}}, \forall \ell \in[0, s]}\{s\}$ ). Note that if $t^{*}=t$ then this implies that $x(t, 0)$ is in $D_{l_{1}}$, hence the result. Assume $t^{*}<t$. This implies that for all $s$ in $\left[0, t^{*}\right]$ we have

$$
\frac{\partial \mathcal{V}}{\partial t}(s, 0)=\frac{\partial V}{\partial x}(x(s, 0)) F_{\omega}(x(s, 0), \omega(s)) \leq \bar{V} .
$$

This gives

$$
\mathcal{V}\left(t^{*}, 0\right) \leq \bar{V} t^{*}+\mathcal{V}(0,0) \leq \bar{V} T_{\min }+m<l_{1} .
$$

Hence $x\left(t^{*}, 0\right)$ is in the interior of $D_{l_{1}}$. It yields that there exists $\varepsilon>0$ such that $x\left(t^{*}+\varepsilon, 0\right)$ is in the interior of $D_{l_{1}}$ which contradicts the fact that $t^{*}$ is an extremum.

$j=1$ This implies that there exists $t_{0}$ in $[0, t]$ such that $\left(t_{0}, 0\right)$ and $\left(t_{0}, 1\right)$ is in $\operatorname{dom}(x)$. Following the first case study, it is possible to show that $x\left(t_{0}, 0\right)$ is in $D_{l_{1}}$. Moreover, we have $x_{p}\left(t_{0}, 1\right)=x_{p}\left(t_{0}, 0\right)$ and, due to 8

$$
x_{c}\left(t_{0}, 1\right)=g\left(x_{c}\left(t_{0}, 0\right), w\left(t_{0}\right)\right) \leq \gamma\left(x_{c}\left(t_{0}, 0\right)\right) .
$$

This implies that $x\left(t_{0}, 1\right) \in D_{l_{1}}^{+}$. Note that $\left[t_{0}, t\right] \mapsto$ $x(s, 1)$ is a continuous mapping with $x\left(t_{0}, 1\right)$ in $D_{l_{2}} \subset$ $D_{n}$. As in the previous case, we define $t^{*}$, the largest time in $\left[t_{0}, t\right]$ such that $x(s, 1)$ is in $D_{n}$ (i.e. $t^{*}=$ $\left.\max _{s \in\left[t_{0}, t\right], x(\ell, 1) \in D_{n}, \forall \ell \in\left[t_{0}, s\right]}\{s\}\right)$. Note that if $t^{*}=t$ then this implies that $x(t, 1)$ is in $D_{n}$, hence the result. Assume $t^{*}<t$. This implies that, for all $s$ in $\left[t_{0}, t^{*}\right]$, it holds

$$
\frac{\partial \mathcal{V}}{\partial t}(s, 1)=\frac{\partial V}{\partial x}(x(s, 1)) F_{\omega}(x(s, 1), \omega(s)) \leq \bar{V} .
$$

This implies

$$
\mathcal{V}\left(t^{*}, 1\right) \leq \bar{V} t^{*}+\mathcal{V}\left(t_{0}, 1\right) \leq \bar{V} T_{\min }+l_{2}<n
$$

Hence $x\left(t^{*}, 1\right)$ is in the interior of $D_{n}$ and following the previous case, we get a contradiction.

This concludes the proof of Lemma 1 .

2) Robustness margin in the compact set:

Lemma 2. (Robustness margin) Under Assumption 3. let $V$ be a function which satisfies (14). Let $D_{n}$ be defined in the previous section. There exist a class $\mathcal{K}$ function $\rho$ and a positive real number $\varepsilon_{r}$ such that, for all $e \in \mathbb{R}^{n_{p}}$, with $|e| \leq \varepsilon_{r}$ and all $\left(x_{p}, x_{c}, \sigma\right)$ in $D_{n}$ the following relations hold.

- If $\left(x_{p}+e, x_{c}, \sigma\right) \in \mathcal{F}_{c} \times[0,+\infty)$

$$
\begin{aligned}
\frac{\partial V}{\partial x}(x) & {\left[\begin{array}{c}
f_{p}\left(x_{p}\right) \\
f_{c}\left(x_{p}+e, x_{c}\right) \\
1-\sigma
\end{array}\right]+\frac{\partial V}{\partial x}(x)\left[\begin{array}{c}
g_{p}\left(x_{p}\right) \\
0 \\
0
\end{array}\right] } \\
& \times \theta_{c}\left(x_{p}+e, x_{c}\right) \leq-\frac{1}{2} V\left(x_{p}, x_{c}\right)+\rho(|e|)
\end{aligned}
$$

- If $\left(x_{p}+e, x_{c}, \sigma\right) \in \mathcal{J}_{c} \times[\lambda,+\infty)$

$V\left(x_{p}, g_{c}\left(x_{p}+e, x_{c}\right), 0\right)-V\left(x_{p}, x_{c}, \sigma\right)$

$$
\leq-\frac{1}{2} \alpha V\left(x_{p}, x_{c}, \sigma\right)+\rho(|e|)
$$

Proof. Employing (14), the set $D_{n}$ being compact and the functions $(F, G, V)$ being continuous, there exists $\varepsilon_{r}$ such that for all $|e| \leq \varepsilon_{r}$ and $\left(x_{p}, x_{c}, \sigma\right)$ in $D_{n}$ then:

- If $\left(x_{p}+e, x_{c}, \sigma\right)$ in $\mathcal{F}_{c} \times[0,+\infty)$,

$$
\frac{\partial V}{\partial x}(x) F(x) \leq-\frac{1}{2} V(x),
$$

- If $\left(x_{p}+e, x_{c}, \sigma\right)$ in $\mathcal{J}_{c} \times[\lambda,+\infty)$

$$
V(G(x))-V(x) \leq-\frac{1}{2} \alpha V(x), \forall x \in \mathcal{J}_{c} \times[0, \lambda]
$$

Consider now the strictly increasing function $\rho:\left[0, \varepsilon_{r}\right) \rightarrow$ $[0,+\infty)$ as the function

$$
\begin{array}{r}
\rho(s) \geq \max \left\{\max _{\left(x_{p}+e, x_{c}, \sigma\right) \in D_{n} \cap \mathcal{F}_{c} \times \mathbb{R}_{+},|e| \leq s} \nu_{1}(x, e),\right. \\
\left.\max _{\left(x_{p}+e, x_{c}, \sigma\right) \in D_{n} \cap \mathcal{J}_{c} \times \mathbb{R}_{+},|e| \leq s} \nu_{2}(x, e)\right\}
\end{array}
$$

where

$$
\begin{aligned}
& \nu_{1}(x, e)=\frac{\partial V}{\partial x}(x)\left(\left[\begin{array}{c}
f_{p}\left(x_{p}\right) \\
f_{c}\left(x_{p}+e, x_{c}\right) \\
1-\sigma
\end{array}\right]-\left[\begin{array}{c}
f_{p}\left(x_{p}\right) \\
f_{c}\left(x_{p}, x_{c}\right) \\
0
\end{array}\right]\right) \\
& \quad+\frac{\partial V}{\partial x}(x)\left(\left[\begin{array}{c}
g_{p}\left(x_{p}\right) \\
0 \\
0
\end{array}\right]\left(\theta_{c}\left(x_{p}+e, x_{c}\right)-\theta_{c}\left(x_{p}, x_{c}\right)\right)\right),
\end{aligned}
$$

and

$$
\nu_{2}(x, e)=V\left(x_{p}, g_{c}\left(x_{p}+e, x_{c}\right), 0\right)-V\left(x_{p}, g_{c}\left(x_{p}, x_{c}\right), 0\right) .
$$

This concludes the proof of Lemma 2.

3) Tuning the high-gain observer: In this subsection we design a high-gain observer for the system (1) in the form

$$
\dot{\hat{x}}_{p}=\Psi_{p}\left(\hat{x}_{p}, y, u\right) .
$$

The function $\Psi_{p}$ is selected to estimate the state $x_{p}$ for initial conditions in the set

$D_{n p}=\left\{x_{p} \in \mathbb{R}^{n_{p}}, \exists\left(x_{c}, \sigma\right) \in \mathbb{R}^{n_{c}} \times \mathbb{R}_{+},\left(x_{p}, x_{c}\right) \in D_{n}\right\}$.

and the control input $u$ is a measurable function such that $|u(t)| \leq \bar{u}$ with $\bar{u}=\max _{x_{p} \in D_{n p},\left(x_{c}, \sigma\right) \in D_{n c}} \theta_{c}\left(x_{p}, x_{c}\right)$ where $D_{n c}=\left\{\left(x_{c}, \sigma\right) \in \mathbb{R}^{n_{c}+1}, \exists x_{p} \in \mathbb{R}^{n_{p}},\left(x_{p}, x_{c}\right) \in D_{n}\right\}$. Moreover, the observer has to be designed such that the estimation error is smaller than the stability margin of the 
controller after $T_{\min }$. More precisely, the observer is such that the set

$$
\left|x_{p}-\hat{x}_{p}\right|<c_{e}, c_{e}:=\min \left\{\rho^{-1}\left(\frac{\alpha n}{3}\right), \varepsilon_{r}\right\}
$$

is reached after $T_{\min }$. With Assumption 4 , we know that the following lemma is satisfied.

Lemma 3. (Tunable observer) There exists a function $\psi$ : $\mathbb{R}^{n_{p}} \times \mathbb{R} \times \mathbb{R}$ and a class $\mathcal{K} \mathcal{L}$ function $\beta$ such that for all $x$ in $D_{n \pi}$ and all $u$ such that $|u(t)| \leq \bar{u}$, if we denote $\left(x_{p}(\cdot), \hat{x}_{p}(\cdot)\right)$ the solution of system $(17)-(18)$ issuing from $\left(x_{p}^{\#}, \hat{x}_{p}^{\#}\right) \in D_{n}$, then for each $t$ such that $x_{p}(s) \in D_{n p}$ for all $s$ in $[0, t]$, we have $\hat{x}_{p}(s)$ is well defined in $[0, t]$ and

1) $\left|x_{p}(s)-\hat{x}_{p}(s)\right| \leq \beta\left(\left|x_{p}^{\#}-\hat{x}_{p}^{\#}\right|, s\right)$;

2) If $t \geq T_{\min },\left|x_{p}(s)-\hat{x}_{p}(s)\right| \leq c_{e}, \forall s \in\left[T_{\min }, t\right]$.

The proof of this result is omitted due to space limitation.

4) Proof of Theorem 2. Consider the positive real number $c_{x}$ obtained in Lemma 1 and the function $k$ obtained in Lemma 3 In the first part of the proof, we show attractivity of the set $\{0\} \times[0,1]$ in $\mathbb{R}^{2 n_{p}+n_{c}} \times[0,1]$ along the solutions to system 9p-10 whose initial conditions are in $\Gamma \times\{0\} \times \mathbb{R}_{+}$.

Let $\left(x_{p}^{\#}, x_{c}^{\#}, \hat{x}_{p}^{\#}, \sigma^{\#}\right)$ be in $\Gamma \times\{0\} \times \mathbb{R}_{+}$and consider a solution $\left(x_{p}, x_{c}, \hat{x}_{p}, \sigma\right)$ whose initial conditions are $\left(x_{p}^{\#}, x_{c}^{\#}, \hat{x}_{p}^{\#}, \sigma^{\#}\right)$ and defined on its time domain denoted dom $\#$.

Note that the system $(9)-(10)$ can be rewritten as the hybrid system $12 p-(13)$ with $\omega=\operatorname{Sat}_{c_{x}}\left(\hat{x}_{p}\right)$ and $\hat{x}_{p}$ is given with the observer (18).

With Lemma 1 and with the $\sigma$ dynamics (persistent flow), we know that there exists $j_{0}$ such that $\left(T_{\min }, j_{0}\right)$ is in $\mathrm{dom}^{\#}$ and for all $(t, j)$ in dom ${ }^{\#}$ with $t \leq T_{\min }$ then $\left(x_{p}(t, j), x_{c}(t, j), \sigma(t, j)\right)$ is in $D_{n}$. Note moreover that this implies that for all $(t, j)$ in dom\# with $t \leq T_{\min }$ then the control input satisfies $|u(t, j)| \leq \bar{u}$. With Lemma 3, we get that the observer state is well defined for all $(t, j)$ in dom ${ }^{\#}$ with $t \leq T_{\min }$. Moreover, for all $(t, j)$ in dom ${ }^{\#}$ with $t \geq T_{\min },\left|x_{p}(t, j)-\hat{x}_{p}(t, j)\right| \leq c_{e}$.

We can now show that for all $(t, j)$ in dom\#,$x(t, j)$ is in $D_{n}$. We will argue by contradiction to prove this assertion. By assuming that it is false, two cases may occur.

1) The solution escapes $D_{n}$ when flowing. Hence, there exists $\left(t_{0}, j_{0}\right)$ in dom ${ }^{\#}$ such that $\left(x_{p}, x_{c}, \sigma\right)\left(t_{0}, j_{0}\right)$ is in $D_{n}$ and for all $\varepsilon>0$ there exists $\delta<\varepsilon$ such that $\left(t_{0}+\delta, j_{0}\right)$ is in dom $\#$ and $\left(x_{p}, x_{c}, \sigma\right)\left(t_{0}+\delta, j_{0}\right)$ is not in $D_{n}$. Note that this implies that $\left(x_{p}, x_{c}\right)\left(t_{0}, j_{0}\right)$ is at the boundary of $D_{n}$. Consequently, this implies $\mathcal{V}\left(t_{0}, j_{0}\right)=n$. Note moreover, that keeping in mind that $\left|x_{p}\left(t_{0}, j_{0}\right)-\hat{x}_{p}\left(t_{0}, j_{0}\right)\right| \leq c_{e} \leq \varepsilon_{r}$ we get employing Lemma $2 \frac{\partial \mathcal{V}}{\partial t}\left(t_{0}, j_{0}\right) \leq-\frac{1}{2} \mathcal{V}\left(t_{0}, j_{0}\right)+\rho\left(c_{e}\right) \leq-\frac{n}{6}$. This implies that the function $s \mapsto \mathcal{V}\left(t_{0}+s, j_{0}\right)$ is strictly decreasing. It contradicts the existence of small $\varepsilon$.

2) The solution escapes $D_{n}$ when jumping. Hence, there exists $\left(t_{0}, j_{0}\right)$ in dom\# such that $\left(x_{p}, x_{c}, \sigma\right)\left(t_{0}, j_{0}\right)$ is in $D_{n}$ and $\left(x_{p}, x_{c}, \sigma\right)\left(t_{0}, j_{0}+1\right)$ is not in $D_{n}$. Since, $\left|x_{p}\left(t_{0}, j_{0}\right)-\hat{x}_{p}\left(t_{0}, j_{0}\right)\right| \leq c_{e} \leq \varepsilon_{r}$, with Lemma 2 , it follows $\mathcal{V}\left(t_{0}, j_{0}+1\right) \leq\left(1-\frac{\alpha}{2}\right) \mathcal{V}\left(t_{0}, j_{0}\right)+\rho\left(c_{e}\right) \leq$ $-\alpha\left(1-\frac{\alpha}{6}\right) n<n$. This is a contradiction with the escape of the solution from $D_{n}$.

Consequently, for all $(t, j)$ in dom\#, we have $x(t, j)$ is in $D_{n}$. Note that the timer forces the $t$ component of the time domain to be unbounded. Hence, thanks to the Lemma 3. $\lim _{t+j \rightarrow+\infty}\left|\hat{x}_{p}(t, j)-x_{p}(t, j)\right|=0$. We get the result employing the triangular structure of the system with the ISS property in $D_{n}$ (i.e. Lemma 2).

To conclude the proof, let us prove the stability property. From the same argument, the set

$$
\begin{aligned}
& S_{v_{0}}=\left\{\left(x_{p}, x_{c}, \hat{x}_{p}, \sigma\right), V\left(x_{p}, x_{c}\right)<v_{0},\right. \\
& \left.\beta\left(\left|x_{p}-\hat{x}_{p}\right|, 0\right)<\rho^{-1}\left(\frac{\alpha v_{0}}{3}\right), \sigma \in\left[0,1+v_{0}\right)\right\}
\end{aligned}
$$

is invariant along solutions for sufficiently small $v_{0}$, is an open set and contains $\{0\} \times[0,1]$ in $\mathbb{R}^{2 n_{p}+n_{c}} \times[0,1]$. Note moreover that for all neighborhoods of $\{0\} \times[0,1]$ in $\mathbb{R}^{2 n_{p}+n_{c}} \times[0,1]$ there exists $v_{0}$ sufficiently small such that $S_{v_{0}}$ is included in it. This allows to conclude stability of the set $\mathbb{R}^{2 n_{p}+n_{c}} \times[0,1]$, and the proof of Theorem 2

\section{REFERENCES}

[1] V. Andrieu and L. Praly. Global asymptotic stabilization for nonminimum phase nonlinear systems admitting a strict normal form. IEEE Transactions on Automatic Control, 53(5):1120-1132, 2008.

[2] V. Andrieu and L. Praly. A unifying point of view on output feedback designs for global asymptotic stabilization. Automatica, 45:17891798, 2009.

[3] V. Andrieu, L. Praly, and A. Astolfi. High-gain observer with uniform in the initial condition finite time convergence. hal-00412585, September 2009.

[4] D. Astolfi and L. Praly. Output feedback stabilization with an observer in the original coordinates for nonlinear systems. In 52nd IEEE Conf. on Dec. Control, Florence, Italy, 2013.

[5] R.W. Brockett. Differential Geometric Control Theory, chapter Asymptotic stability and feedback stabilization, pages 181-191. Birkhauser Boston, 1983.

[6] J.-P. Gauthier, H. Hammouri, and S. Othman. A simple observer for nonlinear systems. Applications to bioreactors. IEEE Transactions on Automatic Control, 37:875-880, 1992.

[7] J.-P. Gauthier and I. Kupka. Deterministic Observation Theory and Applications. Cambridge University Press, 2001.

[8] R. Goebel, R.G. Sanfelice, and A.R. Teel. Hybrid Dynamical Systems. Princeton University Press, 2012.

[9] R. Goebel and A.R. Teel. Solutions to hybrid inclusions via set and graphical convergence with stability theory applications. Automatica, 42:573-587, 2005.

[10] K.H. Johansson, M. Egerstedt, J. Lygeros, and S. Sastry. On the regularization of Zeno hybrid automata. Systems \& Control Letters, 38:141-150, 1999.

[11] G.P. Liu and J.F Patton. Eigenstructure Assignment for Control System Design. Wiley London, 1998.

[12] C. Prieur. Uniting local and global controllers with robustness to vanishing noise. Mathematics of Control, Signals, and Systems, 14(2):143-172, 2001.

[13] C. Prieur and E. Trélat. Robust optimal stabilization of the Brockett integrator via a hybrid feedback. Mathematics of Control, Signals, and Systems, 17:201-216, 2005.

[14] E.D. Sontag. Stability and stabilization: discontinuities and the effect of disturbances. In Nonlinear analysis, differential equations and control (Montreal, QC, 1998), volume 528 of NATO Sci. Ser. C Math. Phys. Sci., pages 551-598. Kluwer Acad. Publ., Dordrecht, 1999.

[15] A.R. Teel and L. Praly. Global stabilizability implies semi-global stabilizability by output feedback. Systems \& Control Letters, 22:313325, 1994. 